\title{
Covariant Approach of the Dynamics of Particles in External Gauge Fields, Killing Tensors and Quantum Gravitational Anomalies ${ }^{\star}$
}

\author{
Mihai VISINESCU
}

Department of Theoretical Physics, National Institute for Physics and Nuclear Engineering, P.O. Box M.G.-6, Magurele, Bucharest, Romania

E-mail: mvisin@theory.nipne.ro

URL: http://www.theory.nipne.ro/ mvisin/

Received February 02, 2011, in final form March 28, 2011; Published online April 05, 2011

doi:10.3842/SIGMA.2011.037

\begin{abstract}
We give an overview of the first integrals of motion of particles in the presence of external gauge fields in a covariant Hamiltonian approach. The special role of Stäckel-Killing and Killing-Yano tensors is pointed out. Some nontrivial examples involving Runge-Lenz type conserved quantities are explicitly worked out. A condition of the electromagnetic field to maintain the hidden symmetry of the system is stated. A concrete realization of this condition is given by the Killing-Maxwell system and exemplified with the Kerr metric. Quantum symmetry operators for the Klein-Gordon and Dirac equations are constructed from Killing tensors. The transfer of the classical conserved quantities to the quantum mechanical level is analyzed in connection with quantum anomalies.
\end{abstract}

Key words: hidden symmetries; Killing tensors; Killing-Maxwell system; quantum anomalies

2010 Mathematics Subject Classification: 81T20; 81T50

\section{Introduction}

Symmetries comprise the most fundamental laws of nature, allowing to find conserved quantities and exact solutions of the equations of motion. The existence of a sufficient number of conserved quantities facilitates the investigation of a given dynamical system. Complete integrability of the Hamiltonian systems is closely related with the separation of variables of the Hamilton-Jacobi equations [1].

The customary conserved quantities originate from geometrical symmetries of the configuration space of the system. These symmetries correspond to Killing (K) vectors representing the isometries of the spacetimes. Beside these symmetries, there exist hidden symmetries generated by higher rank Stäckel-Killing (SK) tensors. The corresponding conserved quantities are quadratic, or, more general, polynomial in momenta connected with symmetries of the complete phase-space.

Another natural generalization of the Killing vectors is represented by the antisymmetric Killing-Yano (KY) tensors [2]. The 'square' of a KY tensor is a SK tensor, but the opposite is not generally true. The symmetries generated by KY tensors are fundamental being involved in many conserved quantities of the quantum systems. For example KY tensors appear in the description of spinning particle systems [3], construction of differential operators which commute with the Dirac operator [4], generation of new exotic supersymmetries [5], and so on.

${ }^{\star}$ This paper is a contribution to the Proceedings of the Conference "Symmetries and Integrability of Difference Equations (SIDE-9)" (June 14-18, 2010, Varna, Bulgaria). The full collection is available at http://www.emis.de/journals/SIGMA/SIDE-9.html 
The conformal extension of the Killing vectors and SK, KY tensors is given by conformal Killing (CK) vectors, conformal Stäckel-Killing (CSK) and conformal Killing-Yano (CKY) tensors respectively. These geometrical objects are involved in the dynamics of the massless particles and are connected with the first integrals of the null geodesics. In recent times the properties of the CKY tensors stimulated much work in generating background metrics with black-holes solutions in higher-dimensional spacetimes (see, e.g. [6]) or interesting geometrical structures [7].

In the study of the dynamics of particles in external gauge fields, the covariant Hamiltonian formulation proposed by van Holten [8] proves to be more adequate involving gauge covariant equations of motion. In particular this approach permits the investigation of the possibility for a higher order symmetry to survive when the electromagnetic interactions are taken into account. This possibility is realized in the Killing-Maxwell (KM) system [9] and an explicit example is provided by the Kerr metric.

An unavoidable problem is the investigation to what extent the classical conservation laws in a curved spacetime could be associated with quantum symmetry operators. It is shown that the CK vectors and CSK tensors do not in general produce quantum operators for the Klein-Gordon equation. In connection with Dirac type operators constructed from KY tensors we discuss the axial anomalies.

The plan of this review paper is as follows. In Section 2 we present the generalized Killing equations in a covariant framework including external gauge fields and scalar potentials. In the next section we produce some examples of conserved quantities of Runge-Lenz type involving external electromagnetic fields. In Section 4 we present various generalizations of the Killing vectors. In Section 5 we analyze the possibility for a hidden symmetry to survive when the electromagnetic interaction is taken into account. We describe the KM system giving a concrete realization in the Kerr spacetime. In Section 6 we examine the quantum version of the hidden symmetries and analyze the gravitational and axial anomalies connected with quantum symmetry operators for Klein-Gordon and Dirac equations. The last section is devoted to conclusions.

\section{Symmetries and conserved quantities}

The classical dynamics of a point charged particle subject to an external electromagnetic field expressed (locally) in terms of the potential 1-form $A_{i}$

$$
F=d A
$$

is derived from the Hamiltonian

$$
H=\frac{1}{2} g^{i j}\left(p_{i}-A_{i}\right)\left(p_{j}-A_{j}\right)+V(x) .
$$

We also added an external scalar potential $V(x)$ for later convenience and $\mathbf{g}$ is the metric of a (pseudo-)Riemmanian $n$-dimensional manifold $\mathcal{M}$.

In terms of the canonical phase-space coordinates $\left(x^{i}, p_{i}\right)$ the conserved quantities commute with the Hamiltonian in the sense of Poisson brackets. The disadvantage of the traditional approach is that the canonical momenta $p_{i}$ and implicitly the Hamilton equations of motion are not manifestly gauge covariant. This inconvenience can be removed using van Holten's receipt [8] by introducing the gauge invariant momenta:

$$
\Pi_{i}=p_{i}-A_{i} .
$$

The Hamiltonian (2) becomes

$$
H=\frac{1}{2} g^{i j} \Pi_{i} \Pi_{j}+V(x),
$$


and equations of motion are derived using the Poisson bracket

$$
\{P, Q\}=\frac{\partial P}{\partial x^{i}} \frac{\partial Q}{\partial \Pi_{i}}-\frac{\partial P}{\partial \Pi_{i}} \frac{\partial Q}{\partial x^{i}}+q F_{i j} \frac{\partial P}{\partial \Pi_{i}} \frac{\partial Q}{\partial \Pi_{j}} .
$$

We mention that in the modified Poisson bracket the momenta $\Pi_{i}$ are not canonical.

A first integral of degree $p$ in the momenta $\Pi$ is of the form

$$
K=K_{0}+\sum_{k=1}^{p} \frac{1}{k !} K_{k}^{i_{1} \ldots i_{k}} \Pi_{i_{1}} \cdots \Pi_{i_{k}},
$$

and it has vanishing Poisson bracket (4) with the Hamiltonian, $\{K, H\}=0$, which implies

$$
\begin{aligned}
& K_{1}^{i} V_{, i}=0, \\
& K_{0}{ }^{, i}+F_{j}{ }^{i} K_{1}^{j}=K_{2}^{i j} V_{, j}, \\
& K_{l}^{\left(i_{1} \ldots i_{l} ; i_{l+1}\right)}+F_{j}{ }^{\left(i_{l+1}\right.} K_{l+1}^{\left.i_{1} \ldots i_{l}\right) j}=\frac{1}{(l+1)} K_{l+2}^{i_{1} \ldots i_{l+1} j} V_{, j} \quad \text { for } \quad l=1, \ldots, p-2, \\
& K_{p-1}^{\left(i_{1} \ldots i_{p-1} ; i_{p}\right)}+F_{j}{ }^{\left(i_{p}\right.} K_{p}^{\left.i_{1} \ldots i_{p-1}\right) j}=0, \\
& K_{p}^{\left(i_{1} \ldots i_{p} ; i_{p+1}\right)}=0 .
\end{aligned}
$$

Here a semicolon denotes the covariant differentiation corresponding to the Levi-Civita connection and round brackets denote full symmetrization over the indices enclosed.

The last equation (6e) is the defining equation of a SK tensor of rank $p$. The SK tensors represent a generalization of the Killing vectors and are responsible for the hidden symmetries of the motions, connected with conserved quantities of the form (5) polynomials in momenta. Indeed, using equation (6e), for any geodesic $\gamma$ with tangent vector $\dot{x}^{i}=p^{i}$

$$
Q_{K}=K_{k}^{i_{1} \ldots i_{k}} p_{i_{1}} \cdots p_{i_{k}}
$$

is constant along $\gamma$. The rest of the equations (6) mixes up the terms of $K$ with the gauge field strength $F_{i j}$ and derivatives of the potential $V(x)$. Several applications using van Holten's covariant framework [8] are given in $[10,11,12,13]$ and a few will be presented in the next section.

\section{$3 \quad$ Explicit examples}

Let us illustrate these general considerations by some nontrivial examples. In what follows the Coulomb potential in a 3 -dimensional Euclidean space $\mathbb{E}^{3}$ will be the basis of the examples superposing different types of electric and magnetic fields. The hidden symmetries which will be found involve SK tensors of rank 2 looking for constants of motion of the form

$$
K=K_{0}+K_{1}^{i} \Pi_{i}+\frac{1}{2} K_{2}^{i j} \Pi_{i} \Pi_{j}
$$

\subsection{Coulomb potential}

To put in a concrete form, we consider the Hamiltonian for the motion of a point charge $q$ of mass $M$ in the Coulomb potential produced by a charge $Q$

$$
H=\frac{M}{2} \dot{\mathbf{x}}^{2}+q \frac{Q}{r} .
$$


We start with (6e) for $p=2$ which is satisfied by a SK tensor of rank 2 . The most general rank 2 SK tensor on 3-dimensional Euclidean space involves some terms which do not contribute nothing of interest for the Coulomb problem and it proved that the following form of the SK tensor is adequate [14]:

$$
K_{2}^{i j}=2 \delta^{i j} \mathbf{n} \cdot \mathbf{x}-\left(n^{i} x^{j}+n^{j} x^{i}\right),
$$

with $\mathbf{n}$ an arbitrary constant vector.

Corresponding to this SK tensor the non-relativistic Coulomb problem admits the RungeLenz vector constant of motion

$$
\mathbf{K}_{2}=\mathbf{p} \times \mathbf{L}+M q Q \frac{\mathbf{x}}{r}
$$

where

$$
\mathbf{L}=\mathbf{x} \times \mathbf{p}
$$

is the angular momentum and $\mathbf{p}=M \dot{\mathbf{x}}$.

\subsection{Constant electric field}

The next more involved example consists of an electric charge $q$ moving in the Coulomb potential in the presence of a constant electric field $\mathbf{E}$. The corresponding Hamiltonian is:

$$
H=\frac{1}{2 M} \mathbf{p}^{2}+q \frac{Q}{r}-q \mathbf{E} \cdot \mathbf{x} .
$$

Again it is adequate to take for the SK tensor of rank 2 the simple form (9) choosing $\mathbf{n}=\mathbf{E}$. Using this form for $K_{2}^{i j}$ after a straightforward working out (6b)

$$
K_{0}=\frac{M q Q}{r} \mathbf{E} \cdot \mathbf{x}-\frac{M q}{2} \mathbf{E} \cdot[\mathbf{x} \times(\mathbf{x} \times \mathbf{E})] .
$$

Concerning equation (6a), it is automatically satisfied by a vector $\mathbf{K}_{1}$ of the form

$$
\mathbf{K}_{1}=\mathbf{x} \times \mathbf{E},
$$

modulo an arbitrary constant factor. This vector $\mathbf{K}_{1}$ contribute to a conserved quantity with a term proportional to the angular momentum $\mathbf{L}$ along the direction of the electric field $\mathbf{E}$.

In conclusion, when a uniform constant electric field is present, the Coulomb system admits two constants of motion $\mathbf{L} \cdot \mathbf{E}$ and $\mathbf{C} \cdot \mathbf{E}$ where $\mathbf{C}$ is a generalization of the Runge-Lenz vector (10):

$$
\mathbf{C}=\mathbf{K}_{2}-\frac{M q}{2} \mathbf{x} \times(\mathbf{x} \times \mathbf{E}) .
$$

\subsection{Spherically symmetric magnetic field}

Another configuration which admits a hidden symmetry is the superposition of an external spherically symmetric magnetic field

$$
\mathbf{B}=f(r) \mathbf{x}
$$

over the Coulomb potential acting on a electric charge $q$. This configuration is quite similar to the Dirac charge-monopole system. 
For $K_{2}^{i j}$ we use again the form (9) and $F_{i j}$ in this case is

$$
F_{i j}=\epsilon_{i j k} B^{k}=\epsilon_{i j k} x^{k} f(r) .
$$

The system of constraint (6) can be solely solved only for a definite form of the function $f(r)$

$$
f(r)=\frac{g}{r^{5 / 2}}
$$

with $g$ a constant connected with the strength of the magnetic field.

With this special form of the function $f(r)$ we get

$$
K_{0}=\left[\frac{M q Q}{r}-\frac{2 g^{2} q^{2}}{r}\right](\mathbf{n} \cdot \mathbf{x})
$$

and

$$
K_{1}^{i}=-\frac{2 g q}{r^{1 / 2}}(\mathbf{x} \times \mathbf{n})^{i} .
$$

Collecting the terms $K_{0}, K_{1}^{i}, K_{2}^{i j}$ the constant of motion (8) becomes

$$
K=\mathbf{n} \cdot\left(\mathbf{K}_{2}+\frac{2 g q}{r^{1 / 2}} \mathbf{L}-2 g^{2} q^{2} \frac{\mathbf{x}}{r}\right)
$$

with $\mathbf{n}$ an arbitrary constant unit vector and $\mathbf{K}, \mathbf{L}$ given by (10), (11) respectively. The angular momentum $\mathbf{L}$ is not separately conserved, entering the constant of motion (12).

\subsection{Magnetic field along a fixed direction}

Another example consists in a magnetic field directed along a fixed unit vector $\mathbf{n}$

$$
\mathbf{B}=B(\mathbf{x} \cdot \mathbf{n}) \mathbf{n},
$$

where, for the beginning, $B(\mathbf{x} \cdot \mathbf{n})$ is an arbitrary function.

Again we are looking for a constant of motion of the form (8) with the SK tensor of rank 2 (9). Equations (6) prove to be solvable only for a particular form of the magnetic field

$$
\mathbf{B}=\frac{\alpha}{\sqrt{\alpha \mathbf{x} \cdot \mathbf{n}+\beta}} \mathbf{n}
$$

with $\alpha$ and $\beta$ two arbitrary constants.

Consequently we get for $K_{0}$ and $K_{1}^{i}$

$$
K_{0}=\frac{M q Q}{r}(\mathbf{x} \cdot \mathbf{n})+\alpha q^{2}(\mathbf{x} \times \mathbf{n})^{2}, \quad K_{1}^{i}=-2 q \sqrt{\alpha \mathbf{x} \cdot \mathbf{n}+\beta}(\mathbf{x} \times \mathbf{n})_{i} .
$$

The final form of the conserved quantity in this case is:

$$
K=\mathbf{n} \cdot\left[\mathbf{K}_{2}+2 q \sqrt{\alpha \mathbf{x} \cdot \mathbf{n}+\beta} \mathbf{L}\right]+\alpha q^{2}(\mathbf{x} \times \mathbf{n})^{2} .
$$

As in the previous example the angular momentum $\mathbf{L}$ is forming part of this constant of motion $K$. 


\subsection{Taub-NUT space and its generalizations}

The four-dimensional Euclidean Taub-NUT geometry is involved in many modern studies in physics (see e.g. [15] and reference therein). The Taub-NUT metric is Ricci-flat self dual on $\mathbb{R}^{4}$ and gives an example of non-trivial gravitational instanton. A Kaluza-Klein monopole was described by embedding the Taub-NUT gravitational instanton into five-dimensional KaluzaKlein theory. Since the classical equations of motion contain the Dirac monopole, a Coulomb potential and a velocity-square dependent term, the Taub-NUT system represents a non-trivial generalization of the Coulomb/Kepler system.

More interestingly, the Kaluza-Klein monopole in classical and quantum mechanics possesses conserved quantities that are analog of the angular momentum and the Runge-Lenz vector of the Kepler problem. Also various generalizations of the Kaluza-Klein monopole system are superintegrable, multiseparable [16].

Let us consider the radially symmetric generalized Taub-NUT metric [17]:

$$
d s^{2}=f(r) \delta_{i j} d x^{i} d x^{j}+h(r)\left(d x^{4}+A_{k} d x^{k}\right)^{2}, \quad i, j, k=1,2,3,
$$

where $r$ is the radial coordinate on $\mathbb{R}^{4}-\{0\}$ and $A_{k}$ is the gauge potential of a Dirac monopole.

For the geodesic motion on the 4-manifold endowed with the metric (13) the canonical momenta conjugate to the coordinates $\left(x^{i}, x^{4}\right)$ are

$$
p_{j}=f(r) \delta_{i j} \frac{d x^{i}}{d t}+h(r)\left(\frac{d x^{4}}{d t}+A_{k} \frac{d x^{k}}{d t}\right) A_{j}, \quad p_{4}=h(r)\left(\frac{d x^{4}}{d t}+A_{k} \frac{d x^{k}}{d t}\right)=q .
$$

Let us remark that the momentum associated with the cyclic variable $x^{4}$ is conserved and interpreted as relative electric charge. Geodesic motion on the 4-manifold projects onto the curved 3 -manifold with metric $g_{i j}=f(r) \delta_{i j}$ augmented with a potential $[17,11]$. In the Hamiltonian (3) the potential is

$$
V(r)=\frac{q^{2}}{2 h(r)},
$$

and accordingly the conserved energy is

$$
E=\frac{\boldsymbol{\Pi}^{2}}{2 f(r)}+\frac{q^{2}}{2 h(r)}
$$

Now the search of conserved quantities of motion in the 3-dimensional curved space in the presence of the potential (14) proceeds as in the previous examples. The zero-order consistency condition (6a) is satisfied for an arbitrary radial potential and entails the conserved angular momentum which involves a typical monopole term

$$
\mathbf{J}=\mathbf{x} \times \mathbf{\Pi}+q \frac{\mathbf{x}}{r} .
$$

Next we search for a Runge-Lenz type vector and for this purpose we start again with the SK tensor of the form (9). The set of equations (6) could be solved in some favorable circumstances. First of all, in the original Taub-NUT space

$$
f(r)=\frac{1}{h(r)}=1+\frac{4 m}{r},
$$

where $m$ is a real parameter, the Runge-Lenz type vector is

$$
\mathbf{K}_{2}=\boldsymbol{\Pi} \times \mathbf{J}-4 m\left(E-q^{2}\right) \frac{\mathbf{x}}{r} .
$$


Another notable case is represented by the Iwai-Katayama generalizations of the Taub-NUT metric [17]

$$
f(r)=\frac{a+b r}{r}, \quad h(r)=\frac{a r+b r^{2}}{1+d r+c r^{2}},
$$

with $a, b, c, d \in \mathbb{R}$. The corresponding Runge-Lenz type vector is

$$
\mathbf{K}_{2}=\mathbf{\Pi} \times \mathbf{J}-\left(a E-\frac{d}{2} q^{2}\right) \frac{\mathbf{x}}{r} .
$$

In both cases, due to the simultaneous existence of the conserved angular momentum (15) and the conserved Runge-Lenz vectors (16) and respectively (17) the motions of the particles are confined to conic sections.

\section{Generalizations of the Killing vectors}

A vector field $X$ on $\mathcal{M}$ is said to be a Killing vector field if the Lie derivative with respect to $X$ of the metric $\mathrm{g}$ vanishes:

$$
L_{X} g=0 .
$$

Killing vector fields can be generalized to CK vector fields [2], i.e. vector fields with a flow preserving a given conformal class of metrics. Furthermore a natural generalization of CK vector fields is given by the CKY tensors [18]. A CKY tensor of rank $p$ on a (pseudo-)Riemmanian manifold $(\mathcal{M}, \mathbf{g})$ is a $p$-form $Y(p \leq n)$ which satisfies:

$$
\left.\nabla_{X} Y=\frac{1}{p+1} X\right\lrcorner d Y-\frac{1}{n-p+1} X^{b} \wedge d^{*} Y
$$

for any vector field $X$ on $\mathcal{M}$, where $\nabla$ is the Levi-Civita connection of $\mathbf{g}, X^{b}$ is the 1 -form dual to the vector field $X$ with respect to the metric, $\lrcorner$ is the operator dual to the wedge product and $d^{*}$ is the adjoint of the exterior derivative $d$. Let us recall that the Hodge dual maps the space of $p$-forms into the space of $(n-p)$-forms. The square of $*$ on a $p$-form $Y$ is either +1 or -1 depending on $n, p$ and the signature of the metric $[19,5]$

$$
* * Y=\epsilon_{p} Y, \quad *^{-1} Y=\epsilon_{p} * Y,
$$

with the number $\epsilon_{p}$

$$
\epsilon_{p}=(-1)^{p} *^{-1} \frac{\operatorname{det} g}{|\operatorname{det} g|}
$$

With this convention, the exterior co-derivative can be written in terms of $d$ and the Hodge star:

$$
d^{*} Y=(-1)^{p} *^{-1} d * Y .
$$

If $Y$ is co-closed in (18), then we obtain the definition of a KY tensor [2]

$$
\left.\nabla_{X} Y=\frac{1}{p+1} X\right\lrcorner d Y .
$$

This definition is equivalent with the property that $\nabla_{j} Y_{i_{1} \ldots i_{p}}$ is totally antisymmetric or, in components,

$$
Y_{i_{1} \ldots i_{p-1}\left(i_{p} ; j\right)}=0 .
$$


The connection with the symmetry properties of the geodesic motion is the observation that along every geodesic $\gamma$ in $\mathcal{M}, Y_{i_{1} \ldots i_{p-1} j} \dot{x}^{j}$ is parallel.

There is also a conformal generalization of the SK tensors, namely a symmetric tensor $K_{i_{1} \ldots i_{p}}=K_{\left(i_{1} \ldots i_{p}\right)}$ is called a conformal Killing (CSK) tensor if it obeys the equation

$$
K_{\left(i_{1} \ldots i_{p} ; j\right)}=g_{j\left(i_{1}\right.} \tilde{K}_{\left.i_{2} \ldots i_{p}\right)}
$$

where the tensor $\tilde{K}$ is determined by tracing the both sides of equation (20). Let us note that in the case of CSK tensors, the quantity (7) is constant only for null geodesics $\gamma$.

These generalizations of the Killing vectors could be related. Let $Y_{i_{1} \ldots i_{p}}$ be a $(\mathrm{C}) \mathrm{KY}$ tensor, then the symmetric tensor field

$$
K_{i j}=Y_{i i_{2} \ldots i_{p}} Y_{j}^{i_{2} \ldots i_{p}},
$$

is a (C)SK tensor and it sometimes refers to this (C)SK tensor as the associated tensor with $Y_{i_{1} \ldots i_{p}}$. That is the case of the Kerr metric [20, 21] or the Euclidean Taub-NUT space [22, 15]. However, the converse statement is not true in general: not all SK tensors of rank 2 are associated with a KY tensor. To wit in the Taub-NUT geometry there are known to exist four KY tensors of which three are covariantly constant. The components of the Runge-Lenz vector (16) are SK tensors which are associated with the KY tensors of the Taub-NUT space. On the other hand, in the case of the Runge-Lenz vector (17) its components are also SK tensors but not associated with KY tensors since the generalized Taub-NUT space [17] does not admit KY tensors [23].

Drawing a parallel between definitions (18) and (19) we remark that all KY tensors are coclosed but not necessarily closed. From this point of view CKY tensors represent a generalization more symmetric in the pair of notions. CKY equation (18) is invariant under Hodge duality that if a $p$-form $Y$ satisfies it, then so does the $(n-p)$-form $* Y$. Moreover the dual of a CKY tensor is a $\mathrm{KY}$ tensor if and only if it is closed.

Let us assume that a CKY tensor of rank $p=2$ is closed $(d Y=0)$ and non-degenerate called a principal $C K Y$ tensor. The principal CKY tensor obeys the following equation [24, 25]

$$
\nabla_{X} Y=X^{b} \wedge \xi^{b}
$$

where $X$ is an arbitrary vector field and

$$
\xi_{i}=\frac{1}{n-1} \nabla_{j} Y_{i}^{j}
$$

Starting with a principal CKY tensor one can construct a tower of CKY tensors formed from external powers $Y^{\wedge k}$ which again are closed CKY tensors. Taking the Hodge dual of these tensors one obtains a set of KY tensors. On the other hand, the vector $\xi_{i}(22)$ obeys the following equation

$$
\xi_{(i ; j)}=-\frac{1}{n-2} R_{l(i} Y_{j)}{ }^{l} .
$$

It is obvious that in a Ricci flat space $\left(R_{i j}=0\right)$ or in an Einstein space $\left(R_{i j} \sim g_{i j}\right), \xi_{i}$ is a Killing vector and we shall refer to it as the primary Killing vector.

\section{$5 \quad$ Killing-Maxwell system}

In this section we shall analyze what is the condition which must be imposed on the gauge fields to preserve the hidden symmetry of the system. Examining the set of coupled equations (6), we observe that the vanishing of the terms $F_{j}{ }^{\left({ }_{l}\right.} K^{\left.i_{1} \ldots i_{l-1}\right) j}$ guarantees that the gauge fields do 
not affect the symmetries of the system. To make things more specific, let us assume that the system admits a hidden symmetry encapsulated in a SK tensor of rank $2, K_{i j}$, associated with a KY tensor $Y_{i j}$ according to (21). The sufficient condition of the electromagnetic field to preserve the hidden symmetry is [12]

$$
F_{k[i} Y_{j]}^{k}=0
$$

where the indices in square bracket are to be antisymmetrized.

It is worth mentioning that this condition appear in many other contexts. For example, using conformal Killing spinors [26] this constraint was resorted to maintain the constant of motion along a null geodesic. Also in the case of the motion of pseudo-classical spinning point particles, relation (23) assures the preservation of the non-generic supersymmetry associated with a KY tensor [27]. In the case of spinor fields on curved space-times this condition is necessary in the construction of Dirac-type operators that commute with the standard Dirac operator [28].

The KM system described by Carter [9] represent an interesting realization of the condition (23). Let us consider the source equation of an electromagnetic field $F_{i j}$ in 4-dimensions

$$
F_{; j}^{i j}=4 \pi j^{i}
$$

and assume that the current $j^{i}$ is a primary Killing vector. Drawing a parallel between equations (22) and (24) we conclude that the electromagnetic field in the KM system is a CKY tensor which, in addition, is a closed 2-form, as in (1). Therefore its Hodge dual

$$
Y_{i j}=* F_{i j},
$$

is a $\mathrm{KY}$ tensor which generates a hidden symmetry (21) associated with it. It is quite simple to verify that $F_{i j} Y_{k}^{j} \sim F_{i j} * F_{k}^{j}$ is a symmetric matrix ( in fact proportional with the unit matrix) making obvious that the constraint (23) is fulfilled.

We complete the description of the KM system observing that the 2-form $Y$ (25) can be written, at least locally, as

$$
Y=* d A,
$$

the form $A$ being usually called a $K Y$ potential.

\subsection{The Kerr metric}

To exemplify the results presented previously, let us consider the Kerr solution to the vacuum Einstein equations which in the Boyer-Lindquist coordinates $(t, r, \theta, \phi)$ has the form

$$
g=-\frac{\Delta}{\rho^{2}}\left(d t-a \sin ^{2} \theta d \phi\right)^{2}+\frac{\sin ^{2} \theta}{\rho^{2}}\left[\left(r^{2}+a^{2}\right) d \phi-a d t\right]^{2}+\frac{\rho^{2}}{\Delta} d r^{2}+\rho^{2} d \theta^{2},
$$

where

$$
\Delta=r^{2}+a^{2}-2 m r, \quad \rho^{2}=r^{2}+a^{2} \cos ^{2} \theta .
$$

This metric describes a rotating black hole of mass $m$ and angular momentum $J=a m$.

As was found by Carter [29], the Kerr space admits the SK tensor

$$
\begin{aligned}
K_{i j} d x^{i} d x^{j}= & -\frac{\rho^{2} a^{2} \cos ^{2} \theta}{\Delta} d r^{2}+\frac{\Delta a^{2} \cos ^{2} \theta}{\rho^{2}}\left(d t-a \sin ^{2} \theta d \phi\right)^{2} \\
& +\frac{r^{2} \sin ^{2} \theta}{\rho^{2}}\left[-a d t+\left(r^{2}+a^{2}\right) d \phi\right]^{2}+\rho^{2} r^{2} d \theta^{2},
\end{aligned}
$$


in addition to the metric tensor $g_{i j}$. This tensor is associated with the KY tensor $[3,30]$

$$
Y=r \sin \theta d \theta \wedge\left[-a d t+\left(r^{2}+a^{2}\right) d \phi\right]+a \cos \theta d r \wedge\left(d t-a \sin ^{2} \theta d \phi\right),
$$

and the dual tensor

$$
* Y=a \sin \theta \cos \theta d \theta \wedge\left[-a d t+\left(r^{2}+a^{2}\right) d \phi\right]+r d r \wedge\left(-d t+a \sin ^{2} \theta d \phi\right),
$$

is a CKY tensor.

The existence of this CKY tensor represents a realization of the KM system and we can verify that the KM four-potential one-form is

$$
A=\frac{1}{2}\left(a^{2} \cos ^{2} \theta-r^{2}\right) d t+\frac{1}{2} a\left(r^{2}+a^{2}\right) \sin ^{2} \theta d \phi .
$$

Finally, the current is to be identified with the primary Killing vector (22)

$$
Y^{l} \partial_{l}:=Y_{; k}^{k l} \partial_{l}=3 \partial_{t} .
$$

\section{Quantum anomalies}

In this section we shall establish a quantum version of the hidden symmetries generated by Killing tensors. We have to be aware of the fact that the classical conserved quantities may not be preserved when we pass to the quantum mechanical level - that is anomalies may occur. For the beginning we shall investigate the quantum symmetry operators for the Klein-Gordon equation. Finally we define conserved operators in the case of Dirac equation and analyze the axial anomalies for the (generalized) Taub-NUT metric.

\subsection{Gravitational anomalies}

Let us now consider the necessary conditions for the existence of constants of motion in the firstquantized system. We start with the classical conserved quantities and take into account that in the quantized system the momentum operator is given by the covariant differential operator $\nabla$ on the manifold $\mathcal{M}$. The corresponding Hamilton operator for a free scalar particle is given by the covariant Laplacian acting on scalars:

$$
\mathcal{H}=\square=\nabla_{i} g^{i j} \nabla_{j}=\nabla_{i} \nabla^{i},
$$

and for a $(\mathrm{C}) \mathrm{K}$ vector we define the quantum symmetry operator

$$
\mathcal{Q}_{V}=K^{i} \nabla_{i}
$$

To consider the analogous condition for the existence of constants of motion in the firstquantized system, we must evaluate the commutator $\left[\square, \mathcal{Q}_{V}\right] \Phi$ acting on some function $\Phi \in$ $\mathcal{C}^{\infty}(\mathcal{M})$, solutions of the Klein-Gordon equation $\square \Phi=0$. The evaluation of this commutator gives:

$$
\left[\mathcal{H}, \mathcal{Q}_{V}\right]=\frac{2-n}{n} K_{k}^{; k i} \nabla_{i}+\frac{2}{n} K_{; k}^{k} \square .
$$

In the case of ordinary Killing vectors, the r.h.s. of this commutator vanishes on the strength of Killing equation, but for CK vectors the situation is different. Since the term $K_{k}{ }^{; k i} \nabla_{i}$ survives for CK vectors, in general the system is affected by quantum anomalies. 
For (C)SK tensors the situation is more intricate. The quantum analogue of classical conserved quantity for a (C)SK tensor of rank 2 is

$$
\mathcal{Q}_{T}=\nabla_{i} K^{i j} \nabla_{j}
$$

On a general manifold this operator does not commute with the Klein-Gordon operator (26) $[7,31]$ :

$$
\begin{aligned}
& {\left[\square, \mathcal{Q}_{T}\right]=2\left(\nabla^{(k} K^{i j)}\right) \nabla_{k} \nabla_{i} \nabla_{j}+3 \nabla_{m}\left(\nabla^{(k} K^{m j)}\right) \nabla_{j} \nabla_{k}} \\
& +\left\{-\frac{4}{3} \nabla_{k}\left(R_{m}^{[k} K^{j] m}\right)+\nabla_{k}\left(\frac{1}{2} g_{m l}\left(\nabla^{k} \nabla^{(m} K^{l j)}-\nabla^{j} \nabla^{(m} K^{k l)}\right)+\nabla_{i} \nabla^{(k} K^{i j)}\right)\right\} \nabla_{j} .
\end{aligned}
$$

We mention that the last term is missing in the corresponding equation in [32].

Some simplifications occur for SK tensor since all the symmetrized derivatives vanish and we end up with a simpler result [32]

$$
\left[\square, \mathcal{Q}_{T}\right]=-\frac{4}{3} \nabla_{i}\left(R_{l}{ }^{[i} K^{j] l}\right) \nabla_{j}
$$

There are a few notable situation for which the quantum system is free of anomalies. Of course if the space is Ricci flat or Einstein, i.e. $R_{i j} \sim g_{i j}$ the r.h.s. of the commutator vanishes. A more interesting and quite unexpected case is represented by SK tensors associated to KY tensors of rank 2 as in (21) [32] a situation which occurs for some spaces [20, 21, 22, 15].

In the case of CSK tensors, practically all terms in (27) survive. Taking into account that the terms are arranged into groups with three, two and just one derivatives it is impossible to have compensations between them.

In conclusion CK vectors and CSK tensors do not in general produce symmetry operators for the Klein-Gordon equation, the quantum system being affected by quantum anomalies.

\subsection{Dirac symmetry operators}

We shall assume that it is possible to define a Dirac spinor structure on the base manifold $\mathcal{M}$. Spinor fields carry irreducible representation of the Clifford algebra and in what follows we shall identify its elements with differential forms. Adopting the convention for the Clifford algebra

$$
e^{a} e^{b}+e^{b} e^{a}=2 g^{a b}
$$

the standard Dirac operator $D$ is written in terms of the spinor connection as

$$
D=e^{a} \nabla_{X_{a}}
$$

A key property of CKY tensors is that one may construct from them symmetry operators for the massless Dirac equation [33, 34]. Let us construct an operator acting on spinors:

$$
L_{Y}=e^{a} Y \nabla_{X_{a}}+\frac{p}{p+1} d Y-\frac{n-p}{n-p+1} d^{*} Y .
$$

In order to construct a Dirac type operator, it proves to be convenient to add to $L_{Y}$ a term proportional to the Dirac operator:

$$
D_{Y}=L_{Y}-(-1)^{p} Y D .
$$

This operator $D_{Y}$ is said to be $R$-(anti)commuting as the graded commutator with the Dirac operator

$$
\left\{D, D_{Y}\right\}_{p}=R D,
$$


where the graded commutator is $[33,34]$

$$
\left\{D, D_{Y}\right\}_{p}=D D_{Y}+(-1)^{p} D_{Y} D .
$$

For the symmetry operator $D_{Y}$ the explicit form of the $R$ operator is

$$
R=\frac{2(-1)^{p}}{n-p+1} d^{*} Y D
$$

Let us remark that the operator $\mathrm{R}$ vanishes when $Y$ is a $\mathrm{KY}$ tensor, otherwise the Dirac type operator $D_{Y}$ gives an on-shell $(D \Psi=0)$ symmetry operator for Dirac operator. The four dimensional case was considered in [35].

\subsection{Axial anomalies}

Having in mind that the KY tensors prevent the appearance of gravitational anomalies for the scalar field, it is natural to investigate whether they play a role also to axial anomalies. Various authors have discussed the electromagnetic and gravitational anomalies in the divergence of the axial-current which is closely related to the existence of zero-eigenvalues of the Dirac operator. The index of the Dirac operator is useful as a tool to investigate topological properties of the space as well as in computing anomalies in quantum field theories.

In what follows we shall consider even-dimensional spaces in which one can define the index of a Dirac operator as the difference in the number of linearly independent zero modes with eigenvalues +1 and -1 under $\gamma_{5}$ :

$$
\operatorname{index}(D)=n_{+}^{0}-n_{-}^{0} .
$$

The commutation relation between the standard and non-standard Dirac operators generated by KY tensor

$$
\left[D_{Y}, D\right]=0
$$

leads to a remarkable result for the index of non-standard Dirac operator:

\section{Theorem 1.}

$$
\operatorname{index}\left(D_{Y}\right)=\operatorname{index}(D) \text {. }
$$

Proof. For a sketch of the proof see [36].

Since we analyzed various properties of the Taub-NUT metric and its generalizations, in what follows we shall also consider the problem of axial anomalies for this space. The remarkable result is that the Taub-NUT metric makes no contribution to the axial anomaly:

Theorem 2. The Dirac operator associated with the standard Taub-NUT metric on $\mathbb{R}^{4}$ does not admit any $L^{2}$ zero modes.

Proof. We sketch the proof [37] observing that the scalar curvature $\kappa$ of the standard TaubNUT metric vanishes. By the Lichnerowicz formula

$$
D^{2}=\nabla^{*} \nabla+\frac{\kappa}{4}=\nabla^{*} \nabla .
$$

Let $\Psi \in L^{2}$ be a solution of $D$, hence $\nabla \Psi=0$. Since a parallel spinor has a constant pointwise norm, it cannot be in $L^{2}$ unless it is 0 , because the volume of $\mathbb{R}^{4}$ with respect to the Taub-NUT metric is infinite. Therefore $\Psi=0$. 
We turn now to the generalized Taub-NUT metric. It has proved in [37] that on the whole generalized Taub-NUT space, although the Dirac operator is not Fredholm, it only has a finite number of null states. In [38] there were found sufficient conditions for the absence of harmonic $L^{2}$ spinors on Iwai-Katayama [17] generalizations of the Taub-NUT space.

Theorem 3. There do not exist $L^{2}$ harmonic spinors on $\mathbb{R}^{4}$ for the generalized Taub-NUT metrics. In particular, the $L^{2}$ index of the Dirac operator vanishes.

Proof. We delegate the proof to [38].

\section{Concluding comments}

The (C)SK and (C)KY tensors are related to a multitude of different topics such as classical integrability of systems together with their quantization, supergravity, string theories, hidden symmetries in higher dimensional black-holes spacetimes, etc.

To conclude let us discuss shortly some problems that deserve a further attention. An obvious extension of the gauge covariant approach to hidden symmetries is represented by the nonabelian dynamics using the appropriate Poisson brackets $[8,10]$. In Section 3 we worked out some examples in an Euclidean 3-dimensional space and restricted to SK tensors of rank 2. More elaborate examples working in a $N$-dimensional curved space and involving SK tensors of higher ranks will be presented elsewhere [39].

Finally, let us mention that the extension of the (C)KY symmetry on spaces with a skewsymmetric torsion is desirable and may provide new insight into the theory of black holes [40].

\section{Acknowledgements}

Support through CNCSIS program IDEI-571/2008 is acknowledged.

\section{References}

[1] Benenti S., Intrinsic characterization of the variable separation in the Hamilton-Jacobi equation, J. Math. Phys. 38 (1997), 6578-6602.

[2] Yano K., Some remarks on tensor fields and curvature, Ann. of Math. (2) 55 (1952), 328-346.

[3] Gibbons G.W., Rietdijk R.H., van Holten J.W., SUSY in the sky, Nuclear Phys. B 404 (1993), 42-64, hep-th/9303112.

[4] Carter B., McLenaghan R.G., Generalized total angular momentum operator for the Dirac equation in curved space-time, Phys. Rev. D 19 (1979), 1093-1097.

[5] Cariglia M., Quantum mechanics of Yano tensors: Dirac equation in curved spacetime, Classical Quantum Gravity 21 (2004), 1051-1077, hep-th/0305153.

[6] Frolov V.P., Hidden symmetries of higher-dimensional black hole spacetimes, Progr. Theor. Phys. Suppl. (2008), no. 172, 210-219, arXiv:0712.4157.

[7] Ianuş S., Visinescu M., Vîlcu G.-E., Conformal Killing-Yano tensors on manifolds with mixed 3-structures, SIGMA 5 (2009), 022, 12 pages, arXiv:0902.3968.

[8] van Holten J.W., Covariant Hamiltonian dynamics, Phys. Rev. D 75 (2007), 025027, 9 pages, hep-th/0612216.

[9] Carter B., Separability of the Killing-Maxwell system underlying the generalized singular momentum constant in the Kerr-Newman black hole metrics, J. Math. Phys. 28 (1987), 1535-1538.

[10] Horváthy P.A., Ngome J.-P., Conserved quantities in anon-abelian monopole field, Phys. Rev. D 79 (2009), 127701, 4 pages, arXiv:0902.0273.

[11] Ngome J.-P., Curved manifolds with conserved Runge-Lenz vectors, J. Math. Phys. 50 (2009), 122901, 13 pages, arXiv:0908.1204. 
[12] Visinescu M., Higher order first integrals of motion in a gauge covariant Hamiltonian framework, Modern Phys. Lett. A 25 (2010), 341-350, arXiv:0910.3474.

[13] Igata T., Koike T., Isihara H., Constants of motion for constrained hamiltonian systems, arXiv:1005.1815.

[14] Crampin M., Hidden symmetries and Killing tensors, Rep. Math. Phys. 20 (1984), 31-40.

[15] Vaman D., Visinescu M., Spinning particles in Taub-NUT space, Phys. Rev. D 57 (1998), 3790-3793, hep-th/9707175.

[16] Marquette I., Generalized Kaluza-Klein monopole, quadratic algebras and ladder operators, arXiv:1103.0374.

[17] Iwai T., Katayama N., On extended Taub-NUT metrics, J. Geom. Phys. 12 (1993), 55-75.

[18] Kashiwada T., On conformal Killing tensor, Natur. Sci. Rep. Ochanomizu Univ. 19 (1968), 67-74.

[19] Kress J., Generalized conformal Killing-Yano tensors: applications to electrodynamics, PhD Thesis, University of Newcastle, 1997.

[20] Floyd R., The dynamics of Kerr fields, PhD Thesis, London University, 1973.

[21] Penrose R., Naked singularities, Ann. New York Acad. Sci. 224 (1973), 125-134.

[22] Gibbons G.W., Ruback R.J., The hidden symmetries of multi-center metrics, Comm. Math. Phys. 115 (1988), 267-300.

[23] Visinescu M., Generalized Taub-NUT metrics and Killing-Yano tensors, J. Phys. A: Math. Gen. 33 (2000), 4383-4391, hep-th/9911126.

[24] Jezierski J., CYK tensors, Maxwell field and conserved quantities for the spin-2 field, Classical Quantum Gravity 19 (2002), 4405-4429, gr-qc/0211039.

[25] Frolov V.P., Hidden symmetries and black holes, J. Phys. Conf. Ser. 189 (2009), 012015, 13 pages, arXiv:0901.1472.

[26] Hughston L.P., Penrose R., Sommers P., Walker M., On a quadratic first integral for the charged particle orbits in the charged Kerr solution, Comm. Math. Phys. 27 (1972), 303-308.

[27] Tanimoto M., The role of Killing-Yano tensors in supersymmetric mechanics on a curved manifold, Nuclear Phys. B 442 (1995), 549-560, gr-qc/9501006.

[28] McLenaghan R.G., Spindel Ph., Quantum numbers for Dirac spinor fields on a curved space-time, Phys. Rev. D 20 (1979), 409-413.

[29] Carter B., Global structure of the Kerr family of gravitational fields, Phys. Rev. 174 (1968), $1559-1571$.

[30] Jezierski J., Łukasik M., Conformal Yano-Killing tensor for the Kerr metric and conserved quantities, Classical Quantum Gravity 23 (2006), 2895-2918, gr-qc/0510090.

[31] Visinescu M., Hidden conformal symmetries and quantum gravitational anomalies, Europhys. Lett. EPL 90 (2010), 41002, 4 pages.

[32] Carter B., Killing tensors quantum numbers and conserved currents in curved spaces, Phys. Rev. D 16 (1977), 3395-3414.

[33] Benn I.M., Charlton P., Dirac symmetry operators from conformal Killing-Yano tensors, Classical Quantum Gravity 14 (1997), 1037-1042, gr-qc/9612011.

[34] Cariglia M., Krtouš P., Kubizňák D., Commuting symmetry operators of the Dirac equation, Killing-Yano and Schouten-Nijenhuis brackets, arXiv:1102.4501.

[35] Kamran N., McLenaghan R.G., Symmetry operators for the neutrino and Dirac fields on curved spacetime, Phys. Rev. D 30 (1984), 357-362.

[36] van Holten J.W., Waldron A., Peeters K., An index theorem for non-standard Dirac operators, Classical Quantum Gravity 16 (1999), 2537-2544, hep-th/9901163.

[37] Moroianu S., Visinescu M., Finiteness of the $L^{2}$-index of the Dirac operator on generalized Euclidean TaubNUT metrics, J. Phys. A: Math. Gen. 39 (2006), 6575-6581, math-ph/0511025.

[38] Moroianu A., Moroianu S., The Dirac operator on generalized Taub-NUT space, arXiv:1003.5364.

[39] Visinescu M., Integrable systems and higher rank Killing tensors, in preparation.

[40] Houri T., Kubizňák D., Warnick C.M., Yasui Y., Generalized hidden symmetries and the Kerr-Sen black hole, J. High Energy Phys. 2010 (2010), no. 7, 055, 33 pages, arXiv:1004.1032. 